\title{
Partial-core transformer design using reverse modelling techniques
}

\author{
M.C.Liew and P.S.Bodger
}

\begin{abstract}
Equivalent circuit components are developed for a partial-core transformer using a new technique called reverse transformer design. Components requiring special consideration include the core-loss resistance, magnetising reactance and the winding leakage reactance. Three sample transformers were built and tested in order to verify and determine the equivalent circuit parameters. A fourth transformer was also designed, built and tested. The measured performance of the transformer confirmed the validity and accuracy of the model.
\end{abstract}

\section{Introduction}

Equivalent circuit modelling has been widely used in designing modern transformers in industry [1-5] and research institutions [6-13]. It has been presented in detail in standard textbooks $[14,15]$. A model is very useful in predicting the performance of a designed transformer. Continuing efforts have been made to improve the accuracy of transformer models [16-21]. Recently, a reverse-design transformer model has been introduced [22]. The specifications are the physical characteristics and dimensions of the windings and core. By manipulating the amount and type of material actually to be used in the construction of the transformer, its performance can be determined. Such an approach lends itself to designing transformers using what is available from suppliers. This is essentially the opposite of the conventional design approach. It offers much flexibility in design and accuracy in predicting performance, compared to the conventional transformer modelling approach [23].

In this paper, the reverse-design method is applied to partial-core transformers. As depicted in Fig. 1, the laminated core occupies the central space only. The windings are wrapped around the core, with the primary winding inside the secondary winding. The yokes and limbs, which usually form the rest of the core in full-core transformers, Fig. 2, are not present. Partial-core transformers are being studied because of their potential use with superconducting windings, where the size of the core can be dramatically reduced, albeit by an increase in winding turns. The combination gives a better magnetisation than a coreless transformer and maintains the leakage flux at an acceptably low level. The combination also means that the overall weight of the partial-core units is significantly reduced, and they are easier to manufacture.

Conventional transformer equivalent circuit components, which are commonly derived, do not readily represent

\footnotetext{
(C) IEE, 2001

IEE Proceedings online no. 20010587

DOI: 10.1049/ip-epa:20010587

Paper received 5th April 2001

The authors are with the Department of Electrical and Electronic Engineering, University of Canterbury, Private Bag 4800, Christchurch, New Zealand
}

partial-core transformers [24]. As a result, modifications are made to these equivalent circuit components to model partial-core transformers. It is thus a goal of this paper to present the partial-core transformer concept for mains frequency and associated modelling, which is sufficiently accurate for practical performance assessment.

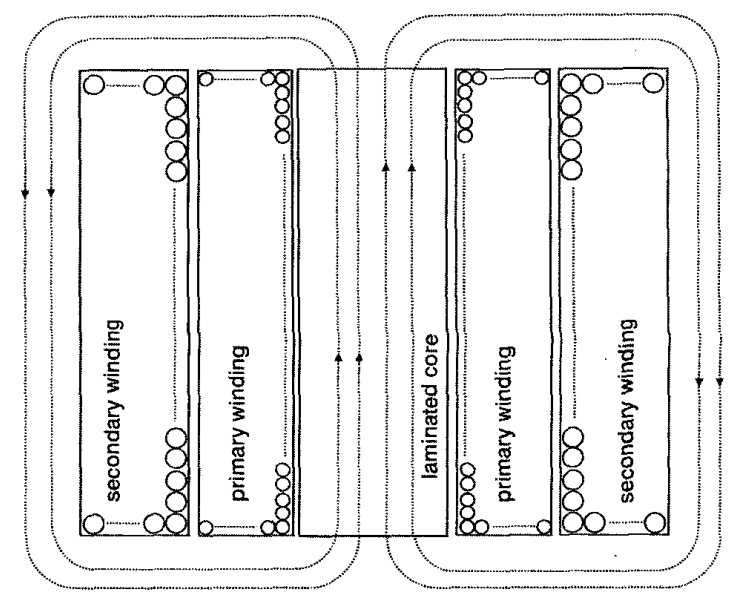

Fig. 1 Cross-sectional view of partial-core transformer

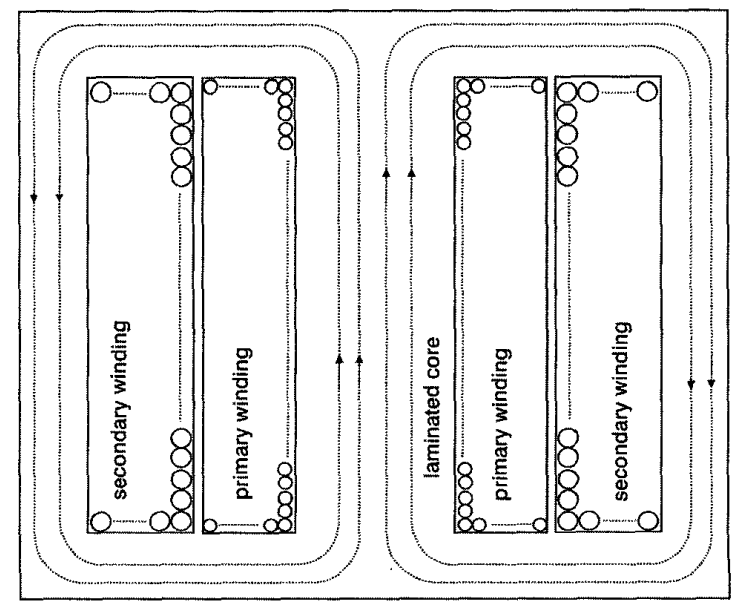

Fig.2 Cross-sectional view of full-core transformer 


\section{Equivalent circuit components formulation}

The equivalent circuit shown in Fig. 3 is often used for supply frequencies [25], where the capacitive effects of insulation do not have a significant impact on the transformer performance. The winding resistances $R_{1}$ and $R_{2}$, which were derived as recorded in [22], are maintained as they are not influenced by the partial-core configuration. However, modifications to the calculation of $X_{m}, X_{12}$ (combination of $X_{1}$ and $X_{2}$ ) and $R_{c}$ are required, as the core is no longer a closed loop. A profile of the partial-core transformer, showing material characteristics and dimensions, is depicted in Fig. 4.

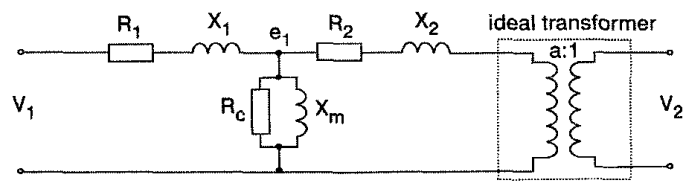

Fig.3 Transformer equivalent circuit, referred to primary winding

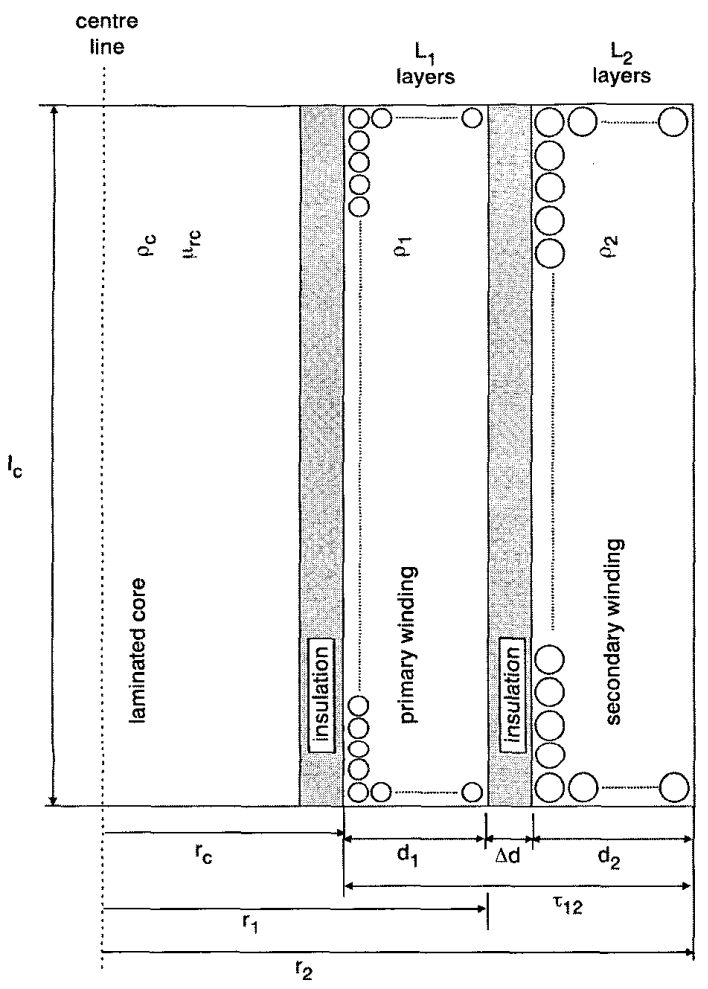

Fig.4 Axial view of transformer showing component dimensions and material properties

\subsection{Magnetising reactance component}

The magnetising current reactance of a full core transformer is calculated as [26]:

$$
X_{m}=\frac{\omega N_{1}^{2} \mu_{o} \mu_{r c} A_{c}}{l_{c}}
$$

where $N_{1}$ is the number of primary turns, $A_{c}$ is the crosssectional area of the core, $l_{c}$ is the length of the core, $\mu_{0}$ is the permeability of free space $=4 \pi \times 10^{-7} \mathrm{Hm}^{-1}, \mu_{r c}$ is the relative permeability of the core, $\omega=2 \pi f$ and $f$ is the input supply frequency.

For a partial-core transformer, the magnetic flux generated by the energised primary winding flows through the core and returns via the air, back to the core. This is depicted in Fig. 5. Therefore a new overall relative permea-

514 bility, which takes into account the magnetic-flux path in the air, has to be determined.

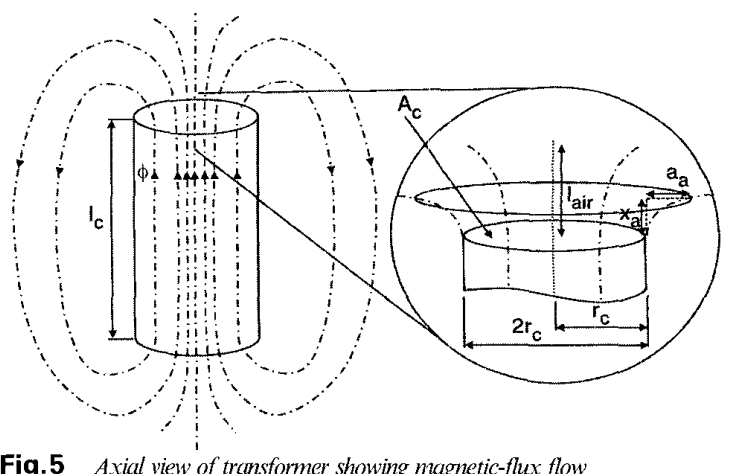

With respect to Fig. 5 , if the flux lines are uniform within the core, then the reluctance of the core is [25]:

$$
\mathcal{R}_{c}=\frac{l_{c}}{\mu_{o} \mu_{r c} A_{c}}
$$

To calculate the reluctance of the air path, it is assumed that the flux density in the air $\left(B_{a i r}=\phi_{a i r} / A_{a i r}\right)$ is only significant near both ends of the core. It is considered negligible elsewhere. Thus, only the air reluctances at both ends of the core are considered.

It is assumed that, at both ends of the core, the flux lines flow into the air and expand as an exponential function, which reaches an asymptotic value of $l_{\text {air }}$. This is an approximation to reality, which allows a simple or naive calculation of the reluctance of the air path of the magnetic circuit. Whereas field analysis using 2D and 3D packages may yield more accurate results, the added complexity in achieving this is over-ridden by the simplicity of the exponential function used, and ultimately the accuracy of the overall performance results. The reluctance is used in the calculation of the magnetising reactance, which has less effect on results than the leakage reactance. The exponential function has the form:

$$
x_{a}=l_{\text {air }}\left(1-\exp \left(\frac{a_{a}}{\vartheta l_{a i r}}\right)\right)
$$

where $x_{a}$ is the vertical distance where the flux travels in the air, $l_{\text {air }}$ is the effective air path length, $a_{a}$ is the horizontal distance from the edge of the core where the flux travels in the air and $\vartheta$ is the partial-core saturation factor.

$\vartheta$ is defined as

$$
\vartheta=k_{\vartheta}\left(\frac{l_{c}}{r_{c}}\right)^{\alpha_{\vartheta}}
$$

where $r_{c}$ is the effective radius of the core $=\sqrt{ }\left(A_{c} / \pi\right), k_{\vartheta}$ is the partial-core saturation constant and $\alpha_{\theta}$ is the partialcore saturation power constant. Therefore, $\vartheta$ is a dimensionless quantity. It determines the rate at which $x_{a}$ reaches its asymptotic value.

Rearranging eqn. 3 gives:

$$
a_{a}=\vartheta l_{\text {air }} \ln \left(\frac{l_{\text {air }}}{l_{\text {air }}-x_{a}}\right)
$$

The flux cross sectional area expands as:

where

$$
A_{a i r}=\pi a_{a i r}^{2}
$$

$$
\begin{aligned}
a_{a i r} & =r_{c}+a_{a} \\
& =r_{c}+\vartheta l_{a i r} \ln \left(\frac{l_{a i r}}{l_{a i r}-x_{a}}\right)
\end{aligned}
$$

IEE Proc.-Electr. Power Appl.. Vol. 148, No. 6, November 2001 
The reluctance of the air can be calculated by integrating over the entire air path length the integral

$$
\mathcal{R}_{a i r}=\int_{0}^{l_{a i r} \rightarrow \infty} \frac{\mathrm{d} x_{a}}{\mu_{o} \mu_{r a} A_{a i r}}
$$

where $\mu_{r a}=$ relative permeability of air $(=1)$.

There is no definite solution for the integral in eqn. 7, so numerical integration has to be performed in order to calculate the air reluctance. The equivalent magnetic circuit of the transformer is given in Fig. 6. The overall reluctance of the transformer is

$$
\mathcal{R}_{T}=\mathcal{R}_{\text {core }}+2 \mathcal{R}_{\text {air }}
$$

The overall relative permeability of the transformer can thus be calculated from:

$$
\mathcal{R}_{T}=\frac{l_{T}}{\mu_{o} \mu_{r} A_{T}}
$$

where $l_{T}$ is the overall flux path length, $l_{c}+2 l_{\text {air }} \approx l_{c}$ (since $\left.l_{c} \gg 2 l_{a \dot{ }}\right)$ and $A_{T}$ is the overall cross sectional area of the transformer $\approx A_{c}$.

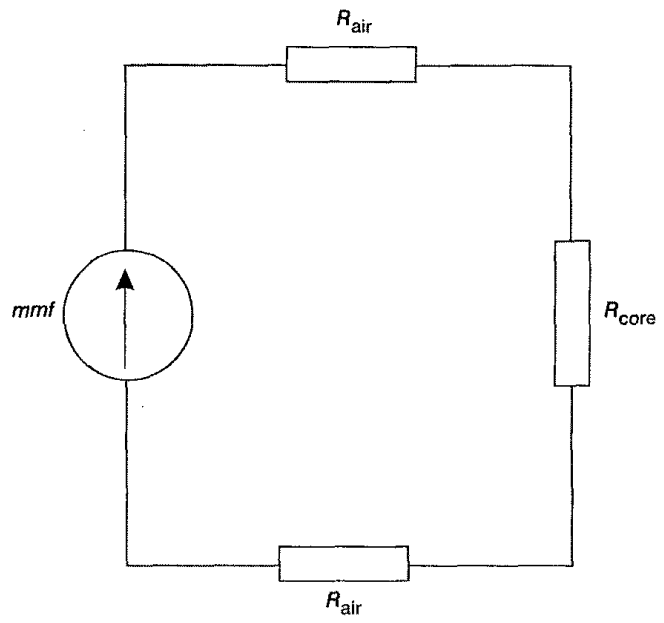

Fig.6 Magnetic circuit of transformer

Rearranging eqn. 9:

$$
\mu_{r T}=\frac{l_{c}}{\mu_{o} \mathcal{R}_{T} A_{c}}
$$

The magnetising reactance is thus

$$
X_{m}=\frac{\omega N_{1}^{2} \mu_{o} \mu_{r T} A_{c}}{l_{c}}
$$

\subsection{Leakage reactance component}

The total leakage reactance of a full-core transformer is calculated from [26]:

$$
X_{12}=\frac{\omega \mu_{o} N_{1}^{2} 4 \tau_{12} \delta^{\prime}}{l_{c}}
$$

where $\tau_{12}$ is the winding thickness factor $=d_{1}+\Delta d+d_{2}$ (see Fig. 4) and $\delta^{\prime}=\left(d_{1}+d_{2}\right) / 3+\Delta d$. A Rogowski factor has been introduced to improve the calculation of the total leakage reactance [27]. The Rogowski factor $(\Gamma)$ is defined as:

$$
\begin{aligned}
& \Gamma \approx 1 \text { when } \beta_{a} \gg 1 \\
& \Gamma<1 \text { when } \beta_{a} \approx 1
\end{aligned}
$$

However, no further specific information has been given on what the exact Rogowski factor should be, given a trans- former with a certain aspect ratio $\left(\beta_{a}\right)$, where

$$
\beta_{a}=\frac{l_{c}}{\tau_{12}}
$$

The conventional equivalent circuit and the concept of leakage reactance for a full-core transformer stem from the difficulty in analysing the behaviour of very tightly coupled coils in terms of self and mutual inductances. The leakagebased approach is well suited to conventional transformers, where the closed magnetic circuit leads to coupling coefficients very close to 1 . This conventional approach is maintained, but modified, for the partial-core transformer, because the closely packed multilayered windings act as guides, which channel the flux up the middle and around the outside such that, in open circuit tests, almost perfect coupling between windings still exists. A similar approach can be used to model induction heaters [28], and has been the basis of computer modelling of a successful commercial line of fluid heaters [29], which uses combined induction and transformer heating and which yielded the conceptual ideas behind the partial-core transformer. Consequently, it is necessary to derive a new expression that governs the Rogowski factor.

The total leakage reactance becomes

$$
X_{12}=\Gamma X_{12}^{\prime}
$$

where $X_{12}^{\prime}$ is the total leakage reactance originally derived in eqn. 12. The Rogowski factor must be redefined. From eqns. 13 and 14, it is evident that the maximum value of $\Gamma$ is 1 :

$$
\Gamma \leq 1
$$

This makes the assumption that for large aspect ratios, i.e. long thin cores, there will be very little leakage. However, the smaller the aspect ratio, the greater the leakage. It has been empirically observed that this is not linearly related.

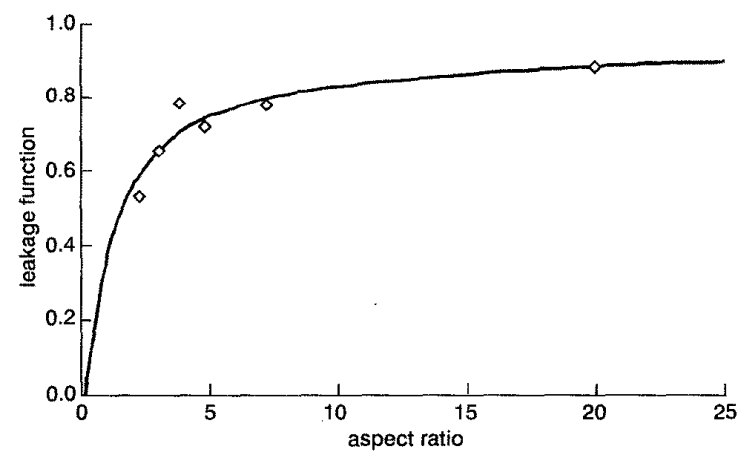

Fig. 7 Exponential leakage function

$\diamond \quad$ measured values

_ _ _ exponential leakage function

It is assumed that $\Gamma$ has an exponential distribution function with respect to $\beta_{a}$, which saturates at $\Gamma=1$, signifying a perfect coupling between windings. This is depicted as the solid line in Fig. 7. The new function, termed the leakage function, thus has the following form:

$$
\Gamma\left(\beta_{a}\right)=1-\exp \left(-\frac{\beta_{a}}{\zeta}\right)
$$

where $\xi$ determines the rate of saturation of the function. The expression given in eqn. 18 is a curve fitted approximation to the empirical observations.

\subsection{Core-loss component}

When calculating the total-core-loss resistance of the partial-core transformer, both the eddy current loss and the 
hysteresis loss components are taken into account [30]. However, it is experimentally observed that the existing Steinmetz's hysteresis loss model [31], which estimates the hysteresis loss component for any full core transformer, is not accurate when applied to partial-core transformers. As a result, modifications have to be made to provide accurate values of these components in the partial-core model.

The hysteresis and eddy current loss components can be represented independently, each by an equivalent resistance [26]. This is shown in Fig. 8. From [30], the hysteresis loss component accounts for $70-80 \%$ of the total transformer core loss.

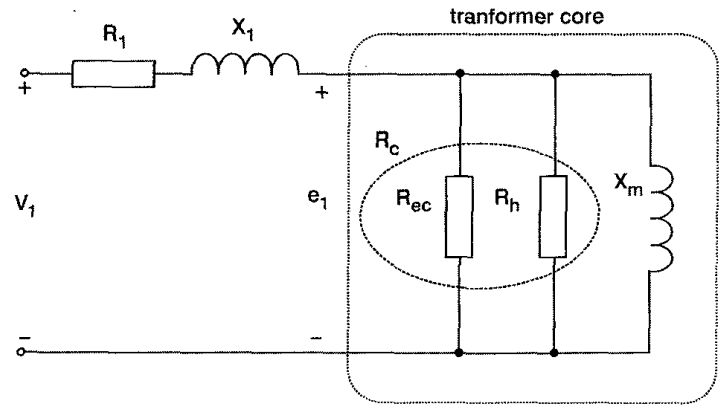

Fig. 8 Hysteresis loss and eddy current loss components equivalent circuit

Steinmetz formulated the hysteresis loss formula for a full iron core transformer as [32]:

$$
\begin{aligned}
P_{h} & =\mathcal{V}_{c} k_{h} f B_{c}^{x} \\
& =\frac{e_{1}^{2}}{R_{h}}
\end{aligned}
$$

where $\mathcal{V}_{c}$ is the volume of the core, $B_{c}$ is the core maximum flux density, $k_{h}$ is a constant dependent on the core material, $x$ is Steinmetz's factor, whose value ranges between 1.8 to 2.5 [25], $e_{1}$ is the emf across the core components (refer to Fig. 8) and $R_{h}$ is the hysteresis resistance.

For the partial-core transformer, a similar expression can be derived. This ignores any effects that the flux remote from the core ends may have in inducing losses in conducting materials in close proximity to the transformer. This is a common problem in induction heating, where judicial design and shielding minimise any significance of this effect. A similar approach has been used for the fluid heating apparatus of [29]. In the end, the numerical value of the core-loss component is dominated by the magnetising reactance, so any error due to proximity effects is not significant.

For the partial-core transformer, $k_{h}$ is defined as:

$$
k_{h}=\left(\mathcal{V}_{c} k_{h p c}\right)^{\alpha_{h p c}}
$$

where $\alpha_{h p c}$ is the partial-core factor and $k_{h p c}$ is the partialcore hysteresis loss constant. Both $k_{h p c}$ and $\alpha_{h p c}$ are constants dependent on the core material. The hysteresis loss for a partial-core transformer thus becomes:

$$
P_{h p c}=\mathcal{V}_{c}^{1+\alpha_{h p c}} k_{h p c}^{\alpha_{h p c}} f B_{c}^{x}
$$

$e_{1}$ is obtained by subtracting the voltage across the primary winding components $\left(R_{1}+\mathrm{j} X_{1}\right)$ from the primary input voltage $V_{1}$.

Eqn. 21 is the general hysteresis loss formula for all partial-core transformers. By substituting $P_{h p c}$ as $P_{h}$ in eqn. $19, R_{h}$ can be calculated. The eddy current resistance $R_{e c}$ is expressed as [26]:

$$
R_{e c}=\frac{N_{1}^{2} A_{c}}{l_{c}} \frac{12 \rho_{c}}{c_{l}}
$$

where $\rho_{c}$ is the operating resistivity of the core and $c_{l}$ is the lamination thickness. The hysteresis resistance $R_{h}$ and the eddy current resistance $R_{e c}$ are in parallel, thus forming the total-core resistance $R_{c}$.

$$
\begin{aligned}
R_{c} & =R_{h} / / R_{e c} \\
& =\frac{R_{h} R_{e c}}{R_{h}+R_{e c}}
\end{aligned}
$$

\section{Determination of the parameters}

To validate the new derivations of the equivalent circuit components for partial-core transformers, three transformers were designed using the reverse approach. Their nominal ratings, physical dimensions and material characteristics are listed in Table 1.

Table 1: Transformer design data

\begin{tabular}{llll}
\hline & \multicolumn{3}{l}{ Transformer } \\
& $\# 1$ & $\# 2$ & $\# 3$ \\
\hline Ratings & & & \\
\hline Primary voltage, V & 120 & 230 & 20 \\
Secondary voltage, V & 30 & 24 & 20 \\
VA rating, VA & 5400 & 4800 & 30 \\
Operating frequency, Hz & 50 & 50 & 50 \\
\hline Core & & & \\
\hline Length, mm & 400 & 150 & 133 \\
Width 1, mm & 31 & 39 & 38 \\
Width 2, mm & 38 & 38 & 44 \\
\hline Primary winding & & & \\
\hline Number of layers & 3 & 11 & 4 \\
Wire diameter, mm & 2.5 & 1.9 & 0.8 \\
\hline Secondary winding & & & \\
\hline Number of layers & 2 & 5 & 4 \\
Wire diameter, mm & 4.5 & 4.5 & 0.8 \\
\hline
\end{tabular}

\section{Table 2: Experimental results for sample transformers}

\begin{tabular}{llll}
\hline $\begin{array}{l}\text { o/c and s/c } \\
\text { test results }\end{array}$ & $\# 1$ & $\# 2$ & $\# 3$ \\
\hline$V_{1 \mathrm{oc}}, \mathrm{V}$ & 120 & 230 & 20 \\
$f_{1 \mathrm{oc},} \mathrm{A}$ & 8.2 & 8.0 & 1.0 \\
$P_{1 \mathrm{oc}}, \mathrm{W}$ & 80 & 183 & 6 \\
$p f_{1 \mathrm{oc}}$ & 0.08 & 0.1 & 0.3 \\
$R_{c}, \Omega$ & 180.9 & 289.0 & 67.3 \\
$X_{m}, \Omega$ & 14.7 & 29.1 & 20.8 \\
\hline$V_{1 \mathrm{sc}}, \mathrm{V}$ & 20 & 143 & 20 \\
$I_{1 \mathrm{sc}}, \mathrm{A}$ & 25.2 & 19.0 & 2.3 \\
$P_{1 \mathrm{sc}}, \mathrm{W}$ & 470 & 1010 & 46 \\
$p f_{1 \mathrm{sc}}$ & 0.95 & 0.37 & 1.0 \\
$R_{\text {wind }}, \Omega$ & 0.74 & 2.77 & 8.5 \\
$X_{\text {leak }} \Omega$ & 0.25 & 6.96 & 1.6 \\
\hline
\end{tabular}

Transformers \#1 and \#2 were designed for high load current applications $(\approx 100 \mathrm{~A})$, each operated at a different supply voltage. Transformer \#3 was a model for a 1:1 isolating transformer. Open circuit and short-circuit tests were carried out on the transformers. For transformers \#1 and \#2 the open circuit tests were carried out at the rated primary input voltages. The short-circuit tests were then 
carried out at current levels lower than the rated currents, owing to the limitations of the test instruments. For transformer \#3, both the open and short-circuit tests were carried out at its rated primary input voltage. The test details are shown in Table 2.

\subsection{Core loss component}

The values of $x, \alpha_{h p c}$ and $k_{h p c}$ in eqns. 19 and 20 were determined experimentally for the same core material used in all three partial-core transformers. Firstly, $R_{h}$ was estimated by rearranging eqn. 23 :

$$
R_{h}=\frac{R_{e c} R_{c}}{R_{e c}-R_{c}}
$$

With the emfs $\left(e_{1}\right)$ measured in the open circuit tests, the hysteresis loss was calculated using eqn. 19. $P_{h}$ was found for each transformer for various input voltage levels. Therefore, the values of $k_{h}$ and $x$ were calculated by solving the following simultaneous equations:

$$
\begin{aligned}
& P_{h(i)}=\mathcal{V}_{c} k_{h} f B_{c(i)}^{x} \\
& P_{h(j)}=\mathcal{V}_{c} k_{h} f B_{c(j)}^{x}
\end{aligned}
$$

where the subscripts $i$ and $j$ denote different input voltage levels. Similarly, the values of $\alpha_{h p c}$ and $k_{h p c}$ in eqn. 20 were calculated by solving, pair by pair, the simultaneous equations of the following three equations:

$$
\begin{aligned}
& k_{h 1}=\left(\mathcal{V}_{c 1} k_{h p c}\right)^{\alpha_{h p c}} \\
& k_{h 2}=\left(\mathcal{V}_{c 2} k_{h p c}\right)^{\alpha_{h p c}} \\
& k_{h 3}=\left(\mathcal{V}_{c 3} k_{h p c}\right)^{\alpha_{h p c}}
\end{aligned}
$$

where the subscripts 1,2 and 3 denote transformers \#1, \#2 and \#3, respectively.

The solutions are

$$
\begin{gathered}
\alpha_{h p c}=\frac{\log \left(k_{h(n)} / k_{h(n+1)}\right)}{\log \left(\mathcal{V}_{c(n)} / \mathcal{V}_{c(n+1)}\right)} \\
k_{h p c}=\frac{k_{h(n)}^{1 / \alpha_{h p c}}}{\mathcal{V}_{c(n)}}
\end{gathered}
$$

where $n=1,2,3$ denotes the transformer number.

Table 3: Computing $\alpha$ and $\boldsymbol{k}_{h p c}$

\begin{tabular}{llll}
\hline & \multicolumn{2}{l}{ Transformer } & \\
& $\# 1$ & $\# 2$ & $\# 3$ \\
\hline$x$ & 1.84 & 1.87 & 1.82 \\
\hline $\begin{array}{l}\text { Solving between } \\
\text { transformers }\end{array}$ & $\# 1$ and \#2 & $\# 2$ and \#3 & $\# 1$ and\#3 \\
\hline$\alpha_{h}$ & -2.46 & -2.62 & -2.47 \\
$k_{h p c}$ & $3.5 \times 10^{3}$ & $3.6 \times 10^{3}$ & $3.5 \times 10^{3}$ \\
\hline
\end{tabular}

Eqns. 30 and 31 were computed for each pair of eqns. 27-29. The results are shown in Table 3. The computed values of $x$ in eqns. 25 and 26 are also shown. The values obtained were very consistent. The average values used in the component modelling are:

$$
\begin{gathered}
x=1.84 \\
\alpha_{h p c}=-2.5 \\
k_{h p c}=3.5 \times 10^{3}
\end{gathered}
$$

\subsection{Magnetising reactance component}

In a similar manner, the values of $\alpha_{\vartheta}$ and $k_{\vartheta}$ were determined for the three transformers. Rearranging eqn. 11, $\mu_{r T}$ of each transformer was calculated from the corresponding measured $X_{m}$. Each respective air reluctance $\mathcal{R}_{\text {air }}$ could then be determined, from which the partial-core saturation factor $\vartheta$ (eqn. 4) was estimated.

The values of $\alpha_{\vartheta}$ and $k_{\vartheta}$ were found by solving, pair by pair, the following three equations:

$$
\begin{aligned}
& \vartheta_{1}=k_{\vartheta}\left(\frac{l_{c 1}}{r_{c 1}}\right)^{\alpha_{\vartheta}} \\
& \vartheta_{2}=k_{\vartheta}\left(\frac{l_{c 2}}{r_{c 2}}\right)^{\alpha_{\vartheta}} \\
& \vartheta_{3}=k_{\vartheta}\left(\frac{l_{c 3}}{r_{c 3}}\right)^{\alpha_{\vartheta}}
\end{aligned}
$$

The solutions are

$$
\begin{gathered}
\alpha_{\vartheta}=\frac{\log \left(\vartheta_{n} / \vartheta_{n+1}\right)}{\log \left(\frac{l_{c(n)} / l_{c(n+1)}}{r_{c(n)} / r_{c(n+1)}}\right)} \\
k_{\vartheta}=\vartheta_{n}\left(\frac{r_{\varepsilon(n)}}{l_{c(n)}}\right)^{\alpha_{\vartheta}}
\end{gathered}
$$

The values obtained are listed in Table 4 . The values obtained were again very consistent. The average values of $\alpha_{\vartheta}$ and $k_{\vartheta}$ used are:

$$
\begin{aligned}
& \alpha_{\vartheta}=0.31 \\
& k_{\vartheta}=2.21
\end{aligned}
$$

Table 4: Estimating $\boldsymbol{\alpha}_{\vartheta}$ and $\boldsymbol{k}_{\vartheta}$

\begin{tabular}{llll}
\hline \multicolumn{4}{c}{ Transformers } \\
& $\# 1$ & $\# 2$ & $\# 3$ \\
\hline$\alpha_{\vartheta}$ & 0.31 & 0.29 & 0.32 \\
$k_{\vartheta}$ & 2.27 & 2.19 & 2.18 \\
\hline
\end{tabular}

\subsection{Leakage reactance component}

Eqn. 18, with the curve of Fig. 7, was supported by actual values from the three sample partial-core transformers. This has subsequently been reinforced from an additional three units not reported on in detail here, but shown in Fig. $7, \zeta$ was found to have a linear relationship with the aspect ratio $\beta_{a}$ :

$$
\zeta=0.4 \beta_{a}+1.59
$$

Thus, from eqn. 18 , the leakage function is:

$$
\Gamma\left(\beta_{a}\right)=1-\exp \left(-\frac{\beta_{a}}{0.4 \beta_{a}+1.59}\right)
$$

\section{Verification of the parameters}

Having developed the equivalent circuit parameters and determined the components within the parameters, the effectiveness of the reverse approach to designing partialcore transformers can be examined. Another transformer, transformer \#4, was designed and built. It also was designed for high-load current applications. Its physical and electrical specifications are listed in Table 5.

The equivalent circuit parameters, referred to the primary winding, and calculated using the new derivations, 
are presented in Table 6. The measured values, as determined by standard open circuit and short circuit tests, are also shown.

Table 5: Transformer design data

\begin{tabular}{ll} 
& Transformer \#4 \\
\hline Ratings & 230 \\
\hline Primary voltage, $\mathrm{V}$ & 25 \\
Secondary voltage, $\mathrm{V}$ & 5000 \\
VA rating, $\mathrm{VA}$ & 50 \\
Operating frequency, $\mathrm{Hz}$ & \\
\hline Core & 195 \\
\hline Length, $\mathrm{mm}$ & 39 \\
Width 1, $\mathrm{mm}$ & 43 \\
Width 2, mm & \\
\hline Primary winding & 9.5 \\
\hline Number of layers & 1.9 \\
Wire diameter, $\mathrm{mm}$ & \\
\hline Secondary winding & 3.5 \\
\hline Number of layers & 4.0 \\
\hline Wire diameter, $\mathrm{mm}$ & \\
\hline
\end{tabular}

Table 6: Calculated and measured equivalent circuit parameters for sample transformer

\begin{tabular}{lll}
\hline $\begin{array}{l}\text { Equivalent circuit } \\
\text { parameters }\end{array}$ & $\begin{array}{l}\text { Transformer } \# 4 \\
\text { calculated }\end{array}$ & measured \\
\hline$R_{c^{\prime}} \Omega$ & 556 & 551 \\
$X_{m} \Omega$ & 45 & 43 \\
$R_{\text {windr }} \Omega$ & 3.8 & 3.5 \\
$X_{\text {leaks }} \Omega$ & 4.8 & 4.8 \\
\hline
\end{tabular}

It can be seen that the core resistances obtained from both the model and the test agree well with each other. Therefore the use of eqn. 21, which calculates the hysteresis loss for partial-core transformers, is confirmed. The use of earlier derivations of $R_{1}$ and $R_{2}$ from previous work [22], which combine to give $R_{w i n d}$, are also validated.

Comparing the values for $X_{m}$, it can be seen that the calculated value is very close to that obtained from the test. Thus the use of $\vartheta$ (eqn. 4), $k_{\vartheta}$ (eqn. 40 ) and $\alpha_{\vartheta}$ (eqn. 41) in eqn. 3 , to find the air reluctance, leading to the computation of $\mu_{r T}$ and $X_{m}$, is justified.

From Table 6 , it can also be seen that there is virtually no difference between the test and calculated values of the total leakage reactance $X_{\text {leak }}$. Thus the validity of the derivation of the new leakage function is corroborated for partial-core transformers.

\section{Table 7: Calculated and measured rated load performance}

\begin{tabular}{lll}
\hline $\begin{array}{l}\text { Performance } \\
\text { parameters }\end{array}$ & \multicolumn{2}{l}{ Transformer $\# 4$} \\
\hline$V_{1}, \mathrm{~V}$ & calculated & measured \\
$I_{1}, \mathrm{~A}$ & 234 & 234 \\
$V_{2}, \mathrm{~V}$ & 21 & 23 \\
$I_{2}, \mathrm{~A}$ & 22 & 25 \\
$P_{1}, \mathrm{~kW}$ & 102 & 109 \\
Efficiency, $\%$ & 59 & 4.2 \\
Regulation, $\%$ & 51 & 60 \\
\hline
\end{tabular}

518
Transformer \#4 was operated at rated conditions to compare calculated and measured values. The results are given in Table 7. The closeness of the calculated and measured results for the transformer in Table 7 further emphasises the formulations derived in the paper.

\section{Conclusions}

Current methods of determining equivalent circuit components for full-core transformers have had notable limitations when applied to partial-core transformers. Consequently, improvements in some of these components are derived and presented using the reverse transformer design method. The dimensions of the core and winding materials are entered, based on what is available. The overall size, ratings and performance of the transformer can then be predicted. The modified components include the core loss resistance, magnetising reactance and the winding leakage reactance.

Three sample transformers have been designed, built and tested to determine the equivalent circuit parameters derived. Components within the parameters have also been determined. A fourth transformer has been designed, built and tested to verify the component models. Significant agreement has been achieved between the values of the transformer equivalent circuit components, as determined through calculation and test. In addition, calculated and measured operational performances of the transformer show good agreement. This opens the way for innovative new designs of transformers with partial-cores, such as welders and superconducting units.

\section{References}

1 HURLEY, W.G., WöLFLE, W.H., and BRESLIN, J.G.: 'Optimized transformer design: inclusive of high-frequency effects', IEEE Trans. Power Electron., 1998, 13, (4), pp. 651-659

2 JUDD, F.F., and KRESSLER, D.R.: 'Design optimization of smal low-frequency power transformers', IEEE Trans. Magn., 1977, MAG13, (4), pp. 1058-1069

3 PETKOV, R.: 'Design issues of high-power high-frequency transformer'. Proceedings of 1995 international conference on Power electronics and drive systems, 1995, Vol. 1, pp. 401-410

4 GILMOUR, A.S.: 'High-power, light-weight power conditioning', IEEE Aerosp. Electron. Syst. Mag., 1991, 6, (12), pp. 33-39

5 O'CONNOR, H.: 'Advanced comprehensive design of line frequency transformers'. Electrical insulation conference and Electrical manufacturing and coil winding conference, 1997, pp. 363-366

6 RUBAAI, A.: 'Computer aided instruction of power transformer design in the undergraduate power engineering class', IEEE Trans. Power Syst., 1994, 9, (3), pp. 1174-1181

7 JEWELL, W.T.: "Transformer design in the undergraduate power engineering laboratory', IEEE Trans. Power Syst, 1990, 5, (2), pp. 499-505

8 SHAHZAD, F., and SHWEHDI, M.H.: 'Human-computer interaction of single/three phase transformer design and performance'. Proceedings of Industrial and commercial power systems technical conference, 1997, pp. 193-196

9 BETANCOURT, R., and TORRE, W.V. 'The use of a design project as an innovative and practical approach to the teaching of power systems', IEEE Trans. Power Syst., 1989, 4, (1), pp. 372-379

10 GRADY, W.M., CHAN, R., SAMOTYJ, M.J., FERRARO, R.J., and BIERSCHENK, J.L.: 'A pc-based computer program for teaching the design and analysis of dry-type transformers', IEEE Trans. Power Syst, 1992,7 (2) pp 709-717

11 POLOUJADOFF, M., and FINDLAY, R.D.: 'A procedure for illustrating the effect of variation of parameters on optimal transformer design', IEEE Trans. Power Syst., 1986, PWRS-1, (4), pp. 202-206

12 MAKRAM, E.B., THOMPSON, R.L., and GIRGIS, A.A.: 'A new laboratory experiment for transformer modeling in the presence of harmonic distortion using a computer controlled harmonic generator', IEEE Trans. Power Syst., 1988, 3, (4), pp. 1857-1863

13 SEMLYEN, A., and HAMADANIZADEH, H.: 'Computational experiments in power systems', IEEE Trans. Power Appar. Syst., 1985, PAS-104, (9), pp. 2290-2295

14 LOWDON, E.: 'Practical transformer design handbook' (McGrawHill, Inc., 2nd edition, 1989)

15 McLYMAN, W.T.: 'Transformer and inductor design handbook' (Dekker, New York, USA, 1988, 2nd edn.) 
16 SATO, T., and SAKAKI, Y.: 'Physical meaning of equivalent loss resistance of magnetic cores', IEEE Trans. Magn., 1990, 26, (5), pp. 2894-2897

17 DE LEON, F., and SEMLYEN, A.: 'Complete transformer model for electromagnetic transients', IEEE Trans. Power Deliv., 1994, 9, (1), pp. 231-239

18 BASAK, A., YU, C.H., and LLOYD, G.: 'Efficient transformer design by computing core loss using a novel approach', IEEE Trans. Magn., 1994, 30, (5), pp. 3725-3728

19 MIRI, S.M., LU, F., and RANDALL, B.E.: 'Design of low-leakage power supply transformers for high-precision electronic instruments', IEEE Trans. Instrum. Meas, 1993, 42, (4), pp. 854-859

20 SARAVOLAC, M.P.: 'Use of advanced software techniques in transformer design'. Proceedings of IEE colloquium on Design technology
of T\&D plant, 1998, pp. 9/1-9/11

21 YAMAGUCHI, H., SATO, Y., and KATAOKA, T.: 'Conceptual design of air-core superconducting transformer for $500 \mathrm{kv}$ cable transmission system', IEE Proc., Gener. Transm. Distrib., 1995, 142, (5), pp. 487-493

22 BODGER, P.S., and LIEW, M.C.: 'Reverse as-built transformer design method', Int. J. Electr. Eng. Educ. (IJEEE), 2002, 39, (1), pp. 52.63

23 BODGER, P.S., and LIEW, M.C.: 'A comparison of conventional and reverse transformer design'. Presented at the Australasian Universities Power Engineering Conference (AUPEC), 2000,
24 ENRIGHT, W., and ARRILLAGA, J.: 'A critique of Steinmetz model as a power transformer representation', Int. J. Elect. Eng.
Educ., 1998, 35, pp. $370-375$

25 PAUL, C.R., NASAR, S.A., and UNNEWEHR, L.E.: 'Introduction to electrical engineering' (Mcgraw-Hill, Inc., Singapore, 1986)

26 SLEMON, G.R.: 'Magnetoelectric devices: transducers, transformers, and machines' (John Wiley and Sons, Inc., USA, 1966)

27 JIANG, Q., and BODGER, P.S.: 'Harmonic response and terminal resonances of high voltage transformers', Int. J. Elec. Engineering Educ., 1991, 28, (2), pp. $144-156$

28 DAVIES, E.J.: 'Conduction and induction heaters, IEE power engineering series II' (Peter Peregrinus Ltd., UK, 1990)

29 BODGER, P.S., WALKER, R.J., and McINNES, I.: 'Significant technology improvement in fluid heating',Proceedings of BNCE/UIE XIII congress on Electricity applications, 1996, pp. 17-24

30 SAY, M.G.: 'Alternating current machines' (Longman Scientific \& Technical, Essex, England, 1983)

31 CONNELLY, F.C.: 'Transformers: their principles and design for light electrical engineers' (Sir Isaac Pitman \& Sons Ltd., London, 1965)

32 McPHERSON, G., and LARAMORE, R.D.: 'An introduction to electrical machines and transformers' (John Wiley and Sons, Inc., New York, USA, 1990, 2nd edn.) 